Editorial

\title{
Artificial Intelligence in Neurointensive Care Unit: A Cautious Leap into Future
}

\author{
Parmod K. Bithal ${ }^{1}$ \\ ${ }^{1}$ Division of Neuroanesthesiology, Department of Anesthesiology \\ and Perioperative Medicine, King Fahad Medical City, Riyadh, Saudi \\ Arabia
}

| Neuroanaesthesiol Crit Care 2020;7:1-2

Artificial intelligence (AI) is the branch of computer science dealing with the simulation of intelligent behavior in computers. ${ }^{1}$ Computers play a key role in almost every aspect of our daily life. In healthcare, computers are an excellent means of storage of patient-related data. The amount of data gleaned electronically from patients admitted in the intensive care units (ICUs) has been growing rapidly every day. Several equipment, such as pressure transducers, infusion pumps, electrocardiography (ECG), pulse oximeters, cardiac output monitors, fluids intake and output monitors, temperature, neurological examination, and mechanical ventilators, interface with computers and store electronic data. Similarly, a wealth of information is recorded from each patient in the ICU, including high-resolution physiological signals, various laboratory tests, and details of medical history in electronic health records (EHRs). ${ }^{2}$ Computerized ICU systems interface, in turn, provide access to hospital database, including demographic, electronic patient records, order entry, laboratory, pharmacy, and radiological systems. To be of use, it is necessary that ICU bedside data must be extracted and organized to become information for clinical decisions. ${ }^{3} \mathrm{AI}$ can assist not only in administering repetitive patient assessment in real time, but also in integrating and interpreting these data source with EHR data, thus potentially enabling more timely and targeted interventions. ${ }^{4,5}$ Closed-loop AI systems can monitor parameters of patients; then, directly treat patients and induce changes in those very parameters that are undergoing monitoring. These systems can make direct real-time adjustments to patient care without any human input. ${ }^{6} \mathrm{AI}$ has proved effective in lowering cost, expanding access, and improving healthcare fields. The application of AI in medicine has been related to the development of AI programs, intended to help the clinician in the making of a diagnosis, adopting therapeutic decisions, and forecasting outcomes. It plays a pivotal role by forewarning impending complications, thereby resulting in a faster response by the clinician. ${ }^{7} \mathrm{AI}$ in an ICU setting could decrease clinicians' as well as nurses' workload,

Address for correspondence Parmod K. Bithal, MD, Division of Neuroanesthesiology, Department of Anesthesiology and Perioperative Medicine, King Fahad Medical City, Riyadh 11525, Saudi Arabia (e-mail: bithal.parmod@gmail.com).

thereby allowing them to focus their attention on critical tasks. It could also augment human decision-making by offering low-cost, high-capacity intelligent data processing.

\section{Utility in Neurocritical Care Units}

Deployment of AI in neurocritical care units (NCCUs) is gradually becoming a reality with the availability of technology. AI systems have made tremendous progress in the realm of analysis of high-resolution neurocritical care data as well as algorithm decision-making. ${ }^{8}$ AI systems will have a significant impact on NCCUs as they are equipped with an array of technologically sophisticated implements to capture and store patient parameters in detail.

NCCUs involve the management of complex neurological patients with inherent limitations of clinical assessment because of the injured brain. Multimodality monitoring generates voluminous data in NCCUs, which can be analyzed with the help of $\mathrm{AI} .^{8}$ Thus, introducing AI in NCCUs will immensely benefit the healthcare providers and patients alike. It is highly likely that traumatic brain injured patients may get the most benefits from AI. It can predict elevation in intracranial pressure (ICP) by advance ICP pulse analysis so that a proactive ICP management could be realized based on these accurate forecasts. ${ }^{9}$ Self-organizing fuzzy logic control (FLC) can administer propofol to provide more stable sedation to forestall the effects of agitation on ICP in traumatic brain injured patients on mechanical ventilation. FLC can compensate for interpatient variation of propofol need. ${ }^{10}$ Furthermore, another algorithm has the ability to predict future mean ICP, which can enable clinicians to identify dangerous trends in ICP early. ${ }^{11}$ Similarly, hypotension, which too adversely impacts outcome of TBI patients, can be predicted beforehand by a Bayesian artificial neural network model. Thus, an early warning of potential hypotensive event before it emerges would allow close monitoring and early clinical assessment to prevent onset of hypotension. ${ }^{12} \mathrm{AI}$ would

Copyright (@2020 Indian Society of Neuroanaesthesiology and Critical Care
License terms

10.1055/s-0040-1701955

ISSN 2348-0548. 
revolutionize management of mechanical ventilation, which is a frequent intervention in neurologically injured patients in NCCU, by ensuring more personalized sedation and analgesia, avoiding unnecessary hyperventilation, and patient readiness for extubation. ${ }^{13}$

Automated detection of seizures from various seizure detecting monitors, prediction of medication response, and even the adjustment of antiepileptic drugs will reduce morbidity in epilepsy patients. ${ }^{14} \mathrm{AI}$ is going to play a greater role in the early management of ischemic stroke and prediction of sepsis in these patients. These patients are generally elderly who may develop delirium in the course of NCCU stay. Overall incidence of delirium is very high in NCCU. ${ }^{15}$ Delirious patients have more complications and may have even worse rehabilitation. AI-enabled data analysis could improve detection of delirium and enable real-time intervention to improve sleep hygiene. ${ }^{16}$

\section{Limitations and Risks}

Of course, using technology to transfer education and healthcare carries some risks. Safeguarding the privacy of patient records must be a top priority because healthcare data are sensitive; so, data security is an important consideration. Appropriate consent must be obtained for data collection; yet many critically ill patients lack sufficient capacity until recovery.

AI may save time, but it cannot listen to a patient. Physical examination will remain important for diagnosis. No one should think that that AI-enabled diagnostic tools will replace doctors or that online learning platforms would supplant teachers, especially when it comes to developing the socioemotional skills.

While AI may enable the designing and development of accurate tools, their introduction must follow careful considerations of real-time clinical utility. The use of AI should be appropriately weighted alongside other sources of available information and should be validated by well-designed prospective studies. Organizations such as Food and Drug Administration (FDA), the Clinical Decision Support Coalition, and Harvard University are offering guidelines on how to move forward with AI in a safe, ethical, and sustainable manner that supports better care while avoiding doomsday scenario if some algorithm goes haywire. Moreover, storing large database in a single location makes the repository a very attractive target for hackers. The full impact of $\mathrm{AI}$ in NCCU cannot be discerned yet as applications still remain in their infancy.

\section{Conflict of Interest}

None declared.

\section{References}

1 Ghahramani Z. Probabilistic machine learning and artificial intelligence. Nature 2015;521(7553):452-459

2 Jalali A, Bender D, Rehman M, Nadkarni V, Natraj C. Clinical systems for patient specific outcomes prediction in intensive care units. Paper presented at: 38th Annual International Conference of the IEEE in Engineering in Medicine and Biology Society (EMBC); Aug 17-20; 2016; Orlando, FL

3 Hanson CW III, Marshall BE. Artificial intelligence applications in the intensive care unit. Crit Care Med 2001;29(2):427-435

4 Vincent JL, Creteur J. Paradigm shifts in critical care medicine: the progress we have made. Crit Care 2015;19(Suppl 3):S10

5 Hirsch LJ. Continuous EEG monitoring in the intensive care unit: an overview. J Clin Neurophysiol 2004;21(5):332-340

6 Rinehart J, Liu N, Alexander B, Cannesson M. Review article: closed-loop systems in anesthesia: is there a potential for closed-loop fluid management and hemodynamic optimization? Anesth Analg 2012;114(1):130-143

7 Vespa PM, Miller C, Hu X, Nenov V, Buxey F, Martin NA. Intensive care unit robotic telepresence facilitates rapid physician response to unstable patients and decreased cost in neurointensive care. Surg Neurol 2007;67(4):331-337

8 Wartenberg KE, Schmidt JM, Mayer SA. Multimodality monitoring in neurocritical care. Crit Care Clin 2007;23(3):507-538

9 Hu X, Xu P, Asgari S, Vespa P, Bergsneider M. Forecasting ICP elevation based on prescient changes of intracranial pressure waveform morphology. IEEE Trans Biomed Eng 2010;57(5):1070-1078

10 Huang SJ, Shieh JS, Fu M, Kao MC. Fuzzy logic control for intracranial pressure via continuous propofol sedation in a neurosurgical intensive care unit. Med Eng Phys 2006;28(7):639-647

11 Zhang F, Feng M, Pan SJ, et al. Artificial neural network based intracranial pressure mean forecast algorithm for medical decision support. Conference proceeding-Annual International Conference of the IEEE. Engineering in Medicine and Biological Society; Jan 1; 2011:7111-7114

12 Donald R, Howells T, Piper I, et al; BrainIT Group. Forewarning of hypotensive events using a Bayesian artificial neural network in neurocritical care. J Clin Monit Comput 2019;33(1):39-51

13 Prasad N, Cheng L-F, Chivers C, Draugelis M, Engelhardt BE. A reinforcement learning approach to weaning of mechanical ventilation in intensive care units. ArXiv170406300 Cs. Available at: http://arxiv.org/abs/1704.06300. Accessed Oct 4, 2019

14 Abbasi B, Goldenholz DM. Machine learning applications in epilepsy. Epilepsia 2019;60(10):2037-2047

15 Nydahl P, Bartoszek G, Binder A, et al. Prevalence for delirium in stroke patients: a prospective controlled study. Brain Behav 2017;7(8):e00748

16 Davoudi A, Malhotra KR, Shickel B, et al. The intelligent ICU for autonomous patient monitoring using pervasive sensing and deep learning. Sci Rep 2019;9(1):8020 